\title{
Optimisation of the ATLAS $b$-tagging algorithms for the 2017-2018 LHC data-taking
}

\author{
F. A. Di Bello on behalf of the ATLAS collaboration* \\ University of Geneva \\ E-mail: fdibello@cern.ch
}

This contribution describes the performance of the ATLAS $b$-tagging algorithms for the 2017-18 data taking at the LHC. Novel taggers based on soft muons from semi-leptonic decays of the $b / c$ hadrons and a Recurrent Neural Network based on track parameters have been integrated into the final high-level discriminant, based on a boosted decision trees. A new training strategy for the optimization of the multivariate techniques in the high- $p_{\mathrm{T}}$ regime will also be presented. Comparisons between data and Monte Carlo simulations and the expected performance for the 2017-18 data taking period will be compared with the former 2016 configuration. The improvements in both, modeling and performance will be discussed.

The European Physical Society Conference on High Energy Physics

5-12 July

Venice, Italy

${ }^{*}$ Speaker. 


\section{Introduction}

The identification of jets containing $b$-hadrons ( $b$-tagging) is key for several physics measurements and searches beyond the Standard Model which can have $b$-jets in the final state.

The discrimination between $b / c$ or light quark and gluon initiated jets is mainly achieved thanks to the long-life time of the $b$ - or $(c)$-hadron. A $b$-hadron with transverse momentum $\left(p_{T}\right)$ of $\sim 50 \mathrm{GeV}$ would travel on average $\sim 4 \mathrm{~mm}$ before decaying. The long life-time can be exploited either by reconstructing the displaced secondary vertex, or by measuring the impact parameters of the tracks reconstructed from the charged decay products of the $b / c$-hadrons.

The ATLAS inner tracker [2] is composed of three main subsystems surrounded by a solenoid providing $2 \mathrm{~T}$ magnetic field. The innermost part of the tracker is composed of four pixels layers. The first layer, also known as Insertable B-Layer (IBL) [3] has been successfully installed in ATLAS before Run 2 to provide improved $b$-tagging performance and more robustness in the measurement of track parameters. The pixel system is followed by 4 layers of silicon micro-strip detectors and, at greater distance, by a Transition Radiation Tracker used to discriminate electrons from charged hadrons.

The $b$-tagging performance presented in this contribution is carried out using $t \bar{t}$ and a broad $Z^{\prime}$ Monte Carlo simulations (MC) corresponding to a $\sqrt{s}=13 \mathrm{TeV}$ proton-proton collisions. The $Z^{\prime}$ sample has been simulated to characterize the high $p_{\mathrm{T}}$ region $\left(p_{\mathrm{T}}>250 \mathrm{GeV}\right)$. In order to produce an approximately flat $p_{\mathrm{T}}$ distribution of the quarks produced in the $Z^{\prime}$ hadronic decays, the crosssection of the hard-scattering process has been modified by applying an event-by-event weighting factor.

To study possible mis-modeling of the $b$-tagging performance evaluated in $\mathrm{MC}$, a $Z \rightarrow \mu \mu+$ jets enriched sample, based on a subset of the data collected by the ATLAS detector in 2016 has been studied. Systematics uncertainty are not included in this preliminary DATA/MC comparison [6].

\section{Algorithms for the identification of heavy flavour jets in ATLAS}

$B$-tagging in ATLAS is performed using underlying algorithms based on the properties of the tracks at the primary vertex and on the reconstruction of displaced vertices. Additional discriminating power can be achieved by exploiting the features of high-quality muon tracks from semileptonic decays of the $b$ - or $c$ - hadron. The output of these algorithms is combined in a multivariate discriminant (MV2). Three main taggers (IP2D, IP3D and RNNIP) are based on the transverse and longitudinal impact parameter (IP), defined as the distance of closest approach to the primary vertex in the $r$ - $\phi$ plane and in the longitudinal plane, respectively. IP2D and IP3D are based on a log-likelihood discriminant which assumes the IPs of the tracks associated to the jet to be uncorrelated. In the 2017-18 configuration a more sophisticated tagger based on Recurrent NN (RNNIP) [4] has been developed to exploit the correlation between the IPs of decay products of the $b$ - or $c$ hadron [6].

Two taggers are based on the reconstruction of displaced vertices: SV1 [5] and JetFitter. SV1 aims at reconstructing a single vertex in a jet. JetFitter, instead, is based on a modified Kalman filter used to reconstruct the full topological decay chain of the $b$-hadrons. In addition, a boosted 
decision trees based on soft muon tracks from semi-leptonic decays of the $b$-hadron (Soft Muon Tagger) has been integrated into the high level-tagger.

The output variables of these algorithms are then combined in MV2. Alternatively to the standard BDT approach a new tagger based on Deep Neural Network has been developed. A comparison between the two approaches is out of the scope of this contribution and can be found elsewhere [6].

\subsection{MV2 and its training strategy}

MV2 is a Boosted Decision Tree (BDT) implemented in the TMVA package [8] which takes as inputs the variables provided by the underlying algorithms.

Three versions of MV2 are studied for the 2017 configuration depending on the choice of the underlying algorithms used as inputs:

- MV2: same inputs variable as in the 2016 configuration, namely: IP2D, IP3D, SV1 and JetFitter.

- MV2mu: addition of the SMT output as input to MV2.

- MV2MuRnn: addition of the RNNIP output as input to MV2Mu.

In the 2016 configuration MV2 was trained on a $t \bar{t}$ sample which has a steeply falling jet- $p_{\mathrm{T}}$ spectrum leading to insufficient statistics for an efficient training of MV2 at high $p_{\mathrm{T}}$. Figure 1 shows the jet- $p_{\mathrm{T}}$ distribution of the $t \bar{t}$ and the broad $Z^{\prime}$ sample designed for the training at high $p_{\mathrm{T}}$. A new training strategy has been employed in order to keep unchanged the performance at low jet$p_{\mathrm{T}}$. A sample made of both $t \bar{t}$, at low $p_{\mathrm{T}}$, and the broad $Z^{\prime}$, at high $p_{\mathrm{T}}$ has been built. Such sample is obtained by including $b$-jets from $t \bar{t}$ if the corresponding $b$-hadron $p_{\mathrm{T}}<250 \mathrm{GeV}$ and from the $Z^{\prime}$ sample if the $b$-hadron $p_{\mathrm{T}}>250 \mathrm{GeV}$. The main reason for this mixing strategy is due to the different gluon-jets composition between the $t \bar{t}$ and the $Z^{\prime}$ samples. By comparing the MV2 output distribution when training on $t \bar{t}$ or $Z^{\prime}$ events it was found that the BDT training process is sensitive to the $u / d / s$ quark- or gluon-jets composition. The fact that the $Z^{\prime}$ sample has a more abundant gluon-jets composition for jet- $p_{\mathrm{T}}<250 \mathrm{GeV}$ leads to suboptimal performance when evaluating on the standard $t \bar{t}$ sample.

For $c$ - and light-flavour jets, the same mixing strategy is applied, moving from $t \bar{t}$ events to $Z^{\prime}$ events for values of the jet $p_{\mathrm{T}}$ above $250 \mathrm{GeV}$. The $p_{\mathrm{T}}$ spectrum for $b$-jet of such sample is shown in Figure 1. This hybrid sample has been successfully used to train the SMT and RNNIP underlying algorithms as well as MV2 [6].

\section{Expected performance for the 2017-2018 LHC data-taking}

In addition to a performant tagger a good DATA/MC agreement has to be foreseen to avoid mis-modelling in the evaluation of the performance which would translate into large scale factors used by physics analysis. In the 2017 configuration a new charge collection model in silicon detectors, the Bischel Model [9], has been used to better describe the MC simulations in data. This modeling improvement also affects the MV2 distribution as shown in Figure 2 where both: the 

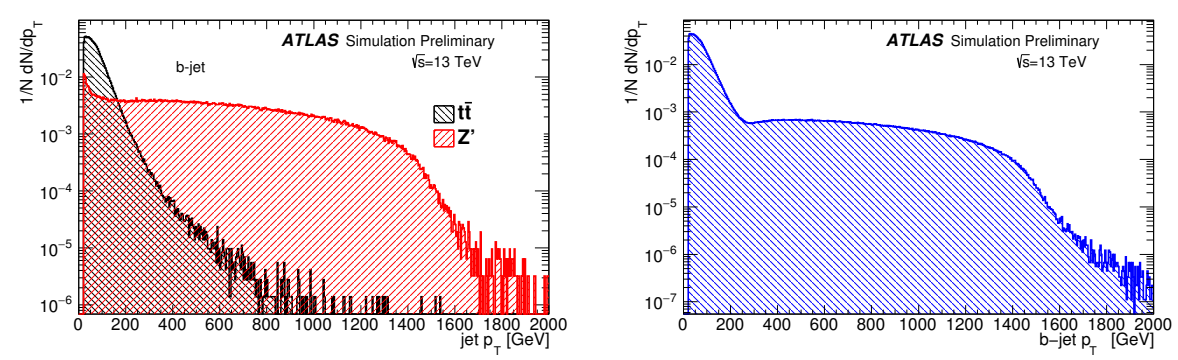

Figure 1: Distribution of the jet transverse momentum of the $Z^{\prime}$ sample compared to that of events from a $t \bar{t}$ sample for $b$-jets (left). $b$-jets $p_{\mathrm{T}}$ distribution of the hybrid sample used for the training of the multivariate-based taggers (right) [6].

2017 and 2016 configurations are shown. From a performance prospective such a model leads to a better estimate of the impact parameter resolution which was found to be underestimated in the past. This propagates into a worsening of the $b$-tagging performance predicted in MC simulations. Figure 3 shows the performance in terms of light and $c$-jets rejections as a function of the jet- $p_{\mathrm{T}}$ for a constant value of $b$-jet efficiency of $77 \%$. Due to the updated estimation of the IP resolution the rejection of light flavoured jets is reduced by around $20 \%$ in the low $p_{\mathrm{T}}$ bin when compared to the 2016 configuration. This loss in performance estimated in MC is expected to retrive scale factors closer to unity for the 2017 configuration. Contrarily, the $c$-jets rejection remains unchanged as the effects of the resolutions are less prominent compared to the semi-leptonically decaying $c$-hadron which can mimic more easily a $b$ quark-initiated jet.

The addition of SMT improves the inclusive performance in the low and medium $p_{\mathrm{T}}$ range for light jet-rejection while not affecting the $c$-rejection as also $c$-hadron may decay semi-leptonically. At high $p_{\mathrm{T}}$, SMT looses its discrimination power as the muon track and the jet-axis are too collimated.

The main improvements in term of light-jets rejection in the high $p_{\mathrm{T}}$ regime is mostly due to the new training strategy and the addition of RNNIP. While at low $p_{\mathrm{T}}$ the inclusion of RNNIP does not bring any improvements in rejecting light-flavoured jet, at high $p_{\mathrm{T}}$, where the other underlying algorithms are suboptimal, the $\mathrm{NN}$ is able to better discriminate $b$-jets by a factor of 3 . For the $c$-jet rejection, instead, MV2MuRnn provides the best discrimination power in the full $p_{\mathrm{T}}$ spectrum due to the fact that the available information is not fully exploited by previous underlying algorithms which do not take into account the differences in the correlation of the IPs of the tracks from $b$ - and $c$-hadron decays. At high $p_{\mathrm{T}}$ this effect is more pronounced leading to an increase of the $c$-rejection of are around $50 \%$.

\section{Conclusion}

Several improvements have been successfully implemented for the 2017 LHC data-taking in ATLAS. Two additional algorithms have been developed and integrated in the final MVA-based tagger. Optimization in the high $p_{\mathrm{T}}$ region has been carried out thanks to a new sample designed for training MV-based taggers. Improvements up to a factor of 3 in light-jets rejection and of around 

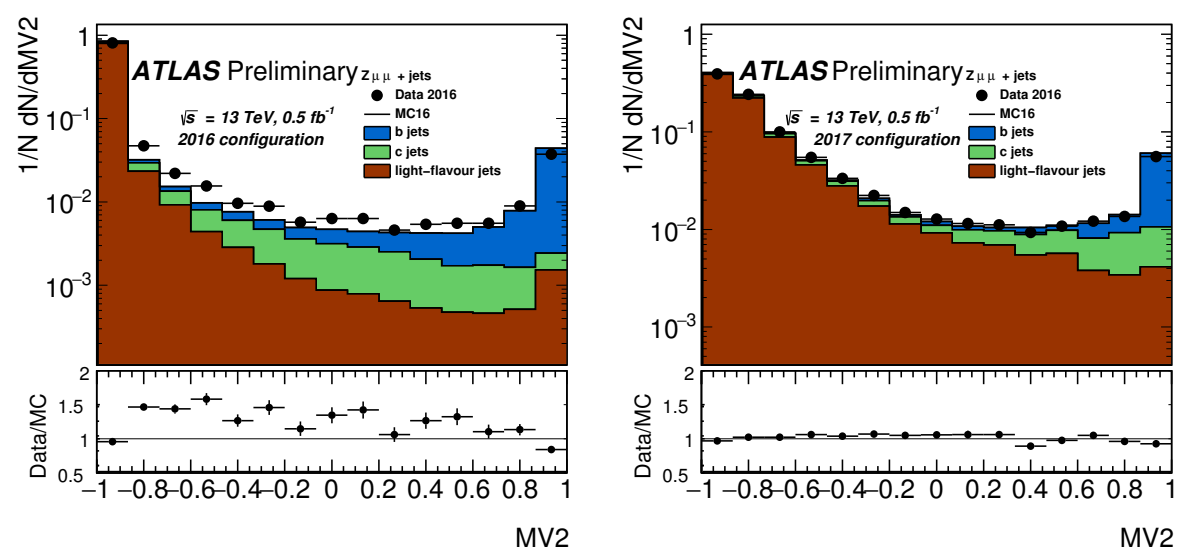

Figure 2: Data-MC comparison of the MV2 output discriminant for $b-, c$-and light-flavour jets using selected $Z \rightarrow \mu^{+} \mu^{-}+$jets-dominated sample for the 2016 (left) and 2017 (right) configuration [6].
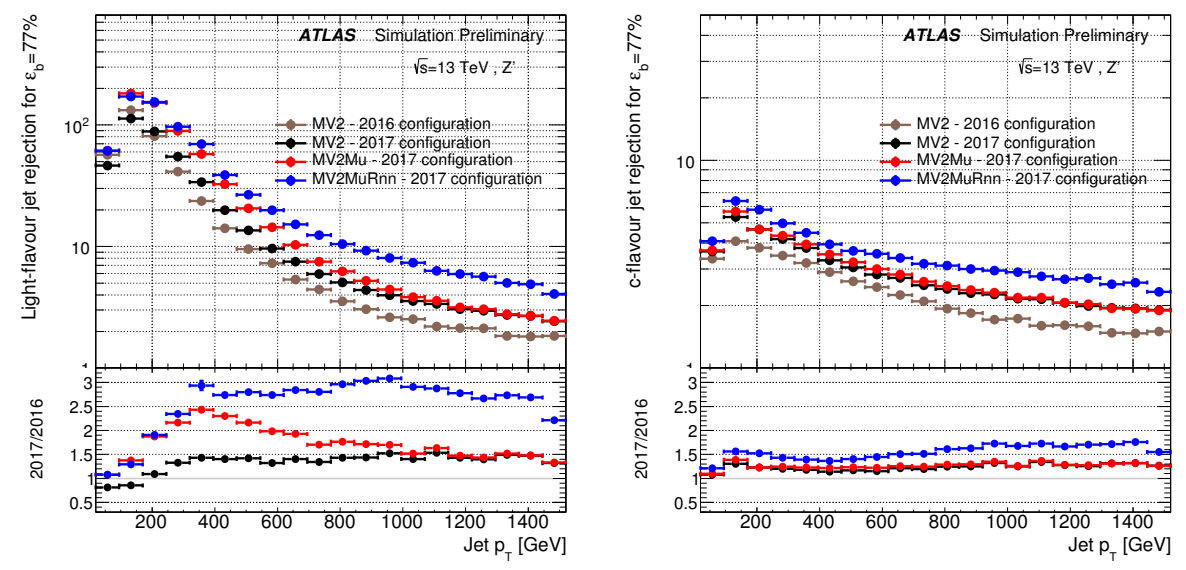

Figure 3: Light-flavour (left) and $c$-jet (right) rejection as a function of the jet- $p_{\mathrm{T}}$ for MV2 in the 2016 configuration (brown markers), MV2 (black markers) in the 2017 configuration, MV2Mu (red markers) and MV2MuRnn (blue markers). The algorithm evaluation is performed on $Z^{\prime}$ events for a flat $b$-jet efficiency of $77 \%$ for each $p_{\mathrm{T}}$ bin. The ratio is calculated with respect to the 2016 configuration [6].

$50 \%$ for $c$-rejection for a $b$-jets efficiency of $77 \%$ are expected. Furthermore, a first DATA/MC comparison shows better agreement for the 2017 configuration. 


\section{References}

[1] ATLAS Collaboration, The ATLAS Experiment at the CERN Large Hadron Collider, JINST 3 (2008) S08003.

[2] ATLAS Collaboration, The ATLAS Inner Detector commissioning and calibration, Eur. Phys. J. C70 (2010) 787.

[3] ATLAS Collaboration, ATLAS Insertable B-Layer Technical Design Report, CERN-LHCC-2010-013. ATLAS-TDR-19.

[4] ATLAS Collaboration, Identification of jets containing b-hadrons with Recurrent Neural Network at the ATLAS experiment, ATLAS-PHYS-PUB-2017-003,

[5] ATLAS Collaboration, Secondary vertex finding for jet flavour identification with the ATLAS detector, ATL-PHYS-PUB-2017-011.

[6] ATLAS Collaboration, Optimisation and performance studies of the ATLAS b-tagging algorithms for the 2017-18 LHC run, ATL-PHYS-PUB-2017-013.

[7] ATLAS Collaboration, Track Reconstruction Performance of the ATLAS Inner Detector at $\sqrt{s}=13$ $\mathrm{TeV}$. ATL-PHYS-PUB-2015-018.

[8] A. Hocker et al., TMVA - Toolkit for Multivariate Data Analysis, PoS ACAT (2007) 040, arXiv.

[9] H. Bichsel, Straggling in thin silicon detectors, Rev. Mod. Phys 60 (1988) 663. 